\title{
A serovar analysis of heterosexual gonorrhoea in Edinburgh 1986-90
}

H Young, A Moyes, J Ross, A McMillan

frequency of isolation of gonococcal serovars from a defined population over a period of 5 years with the aim of defining changes that occur in the variety and frequency of serovars over time and postulates mechanisms to explain the observations.

of such serovars over time.

Methods-All heterosexual patients with a diagnosis of gonorrhoea confirmed on culture between January 1986 and December 1990 had their gonococcal strain serotyped. Temporal changes in the prevalence of gonorrhoea and the serovar of the isolates were analysed.

Results-Isolates of Neisseria gonorrhoeae from 1356 episodes of gonorrhoea were serotyped. Three serovars, Bajk (IB-3/IB-6), Bacejk (IB-1/IB-2) and Aedgkih (IA-1/IA-2), dominated, occurring in two-thirds of all infections. Over the study period Bajk (IB-3/IB-6) and Aedgkih (IA-1/IA-2) isolates declined in frequency in parallel with an overall fall in the prevalence of gonorrhoea but Bacejk (IB-1/IB-2) persisted at a lower but fairly constant level. Despite a fall in the number of gonococcal infections the variety of new serovars being isolated fluctuated.

Conclusions-The ability of some serovars to persist while others decline in incidence may be partially related to antibiotic sensitivities but other factors such as an ability to evade the immune response and transfer of serovars from one population group to another may also be important.

\section{Introduction}

Since the introduction of serotyping for Neisseria gonorrhoeae using monoclonal antibodies it has been possible to divide the gonococcus into subgroups which can be used to study the epidemiology of the organism. ${ }^{1}$ This technique has been used to demonstrate the wide variety of gonococcal serovars present and the rapid dynamic change that occurs within any one area. ${ }^{23}$ It has also been noted that different geographical areas may have marked differences in the serovars which are dominant despite being within reasonably close proximity. 45

Although this epidemiological information is available for individual regions at one point in time there is much less known about the changes in serovars over time and, more importantly, the factors responsible for observed changes. This study follows the

\section{Methods}

All heterosexual patients (women admitting to male contacts and men admitting to female contacts only) with a diagnosis of gonorrhoea who presented to the Department of Genitourinary Medicine, Edinburgh Royal Infirmary between January 1986 and December 1990 were included in the study. The diagnosis of gonorrhoea was made on the basis of culture of $N$. gonorrhoeae on modified New York City culture medium from the urethra, rectum, endocervix and/or throat. All male patients attending the Department had a single urethral swab taken whilst female patients had urethral, rectal and endocervical swabs cultured on two separate occasions in order to diagnose or exclude gonorrhoea. Throat cultures were performed in all partners of patients with gonorrhoea and when the history indicated that this site had been placed at risk.

Gonococcal isolates were identified on the basis of biochemical and immunological tests and serotyping was performed using the Swedish panel of Genetic Systems reagents as has been described previously. ${ }^{6}$ Serovars were designated according to the descriptive nomenclature and later translated into the likely equivalent serovars based on the more widely used American Genetic Systems panel and numerical nomenclature. ${ }^{78}$

The total number of isolates each quarter was noted and further subdivided into isolations from male and female patients. The number of infections due to the three most commonly isolated serovars (Bajk (IB-3/IB-6), Bacejk (IB-1/IB-2) and Aedgkih (IA-1/IA-2)) were calculated separately for each quarter. The number of different serovars present within each quarter was also counted and the location of where the infection was acquired noted.

Statistics Statistical analysis was performed using Spearman's Rank Correlation coefficient on the Minitab PC software package.

\section{Results}

The number of infections in men and women is shown in table 1: 1356 episodes of heterosexually acquired gonorrhoea were identified 
Table 1 Number of male and female heterosexually acquired gonococcal infections in Edinburgh 1986-90

\begin{tabular}{llcc}
\hline & & Male & Female \\
\hline 1986 & 1st Quarter & 83 & 45 \\
& 2nd Quarter & 79 & 55 \\
& 3rd Quarter & 83 & 53 \\
& 4th Quarter & 66 & 54 \\
1987 & 1st Quarter & 59 & 43 \\
& 2nd Quarter & 60 & 39 \\
& 3rd Quarter & 49 & 36 \\
1988 & 4th Quarter & 52 & 37 \\
& 1st Quarter & 38 & 27 \\
& 2nd Quarter & 33 & 23 \\
& 3rd Quarter & 39 & 27 \\
1989 & 4th Quarter & 24 & 21 \\
& 1st Quarter & 15 & 14 \\
& 2nd Quarter & 18 & 16 \\
& 3rd Quarter & 15 & 10 \\
1990 & 4th Quarter & 15 & 13 \\
& 1st Quarter & 13 & 14 \\
& 2nd Quarter & 16 & 17 \\
& 3rd Quarter & 18 & 15 \\
Total & 4th Quarter & 14 & 8 \\
\hline
\end{tabular}

over the five year study period. The number of episodes of heterosexually acquired gonorrhoea fell from 518 in 1986 to 115 in 1990. Thirteen protein IA serovars accounted for 711 $(52 \%)$ of infections while 31 protein IB serovars accounted for $645(48 \%)$. There were 10 IA serovars and 20 IB serovars isolated from women compared with 12 IA and 28 IB serovars from men. The higher number of serovars in male infections is in keeping with the greater number of infections observed in men (table 1). As shown in fig 1 the combined number of different IA and IB serovars has decreased from a total of 14 in the first quarter of 1986 to seven during the last quarter of 1990 . In spite of the overall trend towards a decrease in the number of different serovars there is a degree of fluctuation particularly in the number of IB serovars isolated each quarter. This could be due to importation of strains.
The geographical area where the infections were acquired is given in table 2 . As shown, only $64 \%$ of infections were acquired within Edinburgh and the surrounding Lothian region: other areas of Scotland $(23 \%$ of infections) provided the main source of imported strains with a less significant contribution from the rest of the UK $(4 \%)$ and abroad (5\%). The geographical area of acquisition of infection did not vary over the study period.

Three serovars Bajk (IB-3/IB-6), Bacejk (IB-1/IB-2) and Aedgkih (IA-1/IA-2) accounted for $69 \%$ (934) of the infections over the 5 year study period. The correlation coefficients relating to these three serovars and the group of "other" serovars to the overall prevalence of gonorrhoea and to the sex of the patients from whom the serovars were isolated are given in table 3. Each of the serovars occurred with similar frequency in men and women, as would be expected in the case of heterosexually acquired gonorrhoea. Whereas the prevalence of Baik (IB-3/IB-6) and Aedgkih (IA-1/IA-2) and "other" serovars correlates well with the fall in total gonorrhoea serovar, Bacejk (IB-1/IB-2) did not.

\section{Discussion}

Public awareness of the risk of contracting sexually transmitted diseases increased following the recognition of Human Immunodeficiency Virus (HIV) infection and there has

Table 2 Geographical origin of heterosexually acquired gonococcal infections in Edinburgh 1986-90

\begin{tabular}{lrr}
\hline Edinburgh & 788 & $(58 \%)$ \\
Rest of Lothian & 83 & $(6 \%)$ \\
Rest of Scotland & 311 & $(23 \%)$ \\
Rest of UK & 55 & $(4 \%)$ \\
Not UK & 71 & $(5 \%)$ \\
Unknown & 48 & $(4 \%)$ \\
\hline
\end{tabular}

Figure 1 Number of different protein $1 A$ and protein $1 B$ serovars isolated over time.

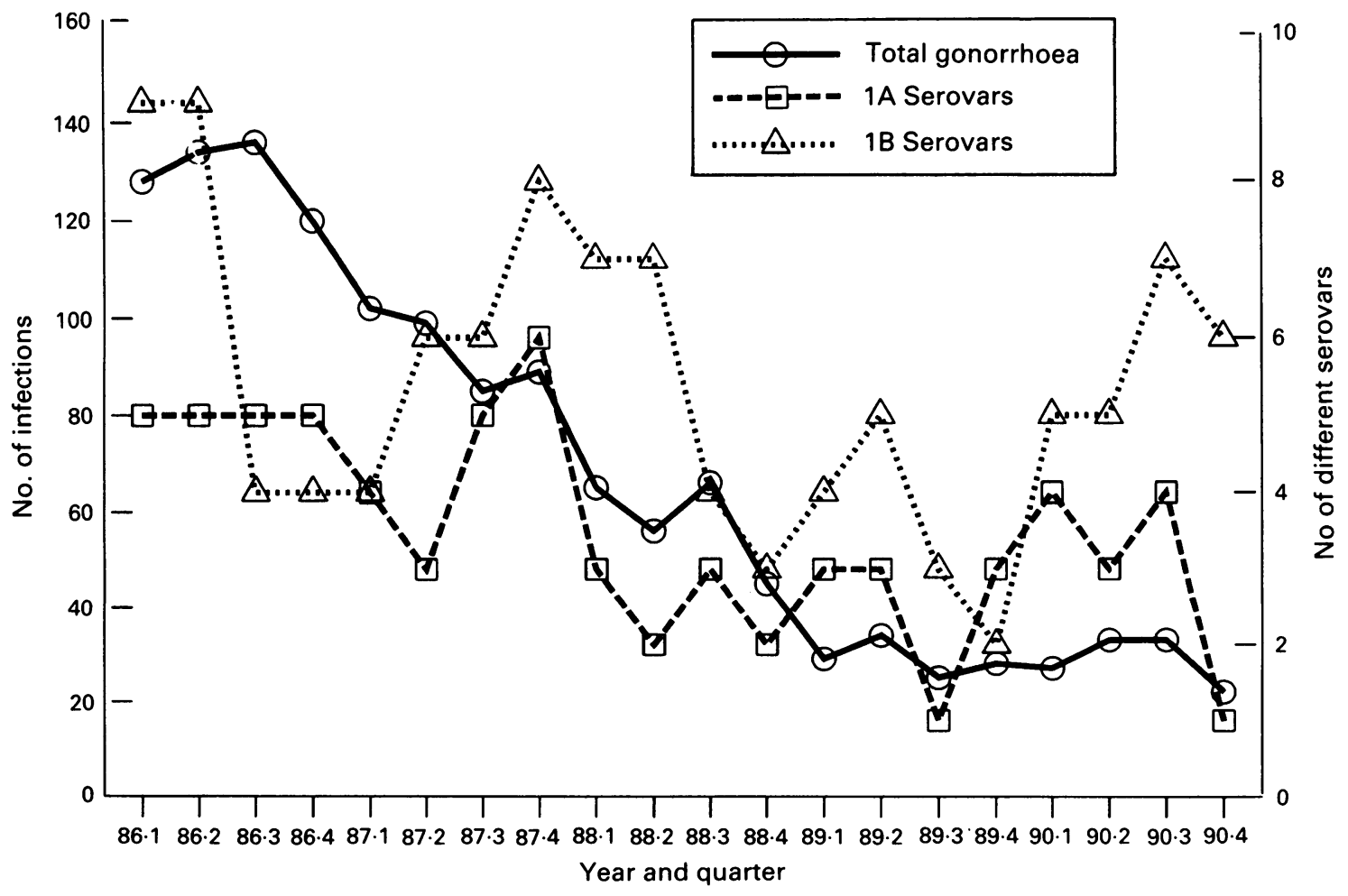


Table 3 Prevalence of serovars correlated with total gonorrhoea and sex of patient

\begin{tabular}{lll}
\hline & $\begin{array}{l}\text { Total gonorrhoea } \\
\text { correlation coefficient }\end{array}$ & $\begin{array}{l}\text { Sex of patient }{ }^{\dagger} \\
\text { correlation coefficient }\end{array}$ \\
\hline Bajk (IB-3/IB-6) & 0.87 & 0.93 \\
Bacejk (IB-1/IB-2) & $0.42^{\star}$ & $0 \cdot 81$ \\
Aedgkih (IA-1/IA-2) & 0.94 & 0.84 \\
"Other" serovars & 0.78 & 0.78 \\
\hline
\end{tabular}

^No significant correlation between Bacejk and total gonorrhoea-all other correlations significant $(p<0.001)$.

† Significant correlation in the occurrence of each serovar between male and female patients $(p<$ $0.001)$.

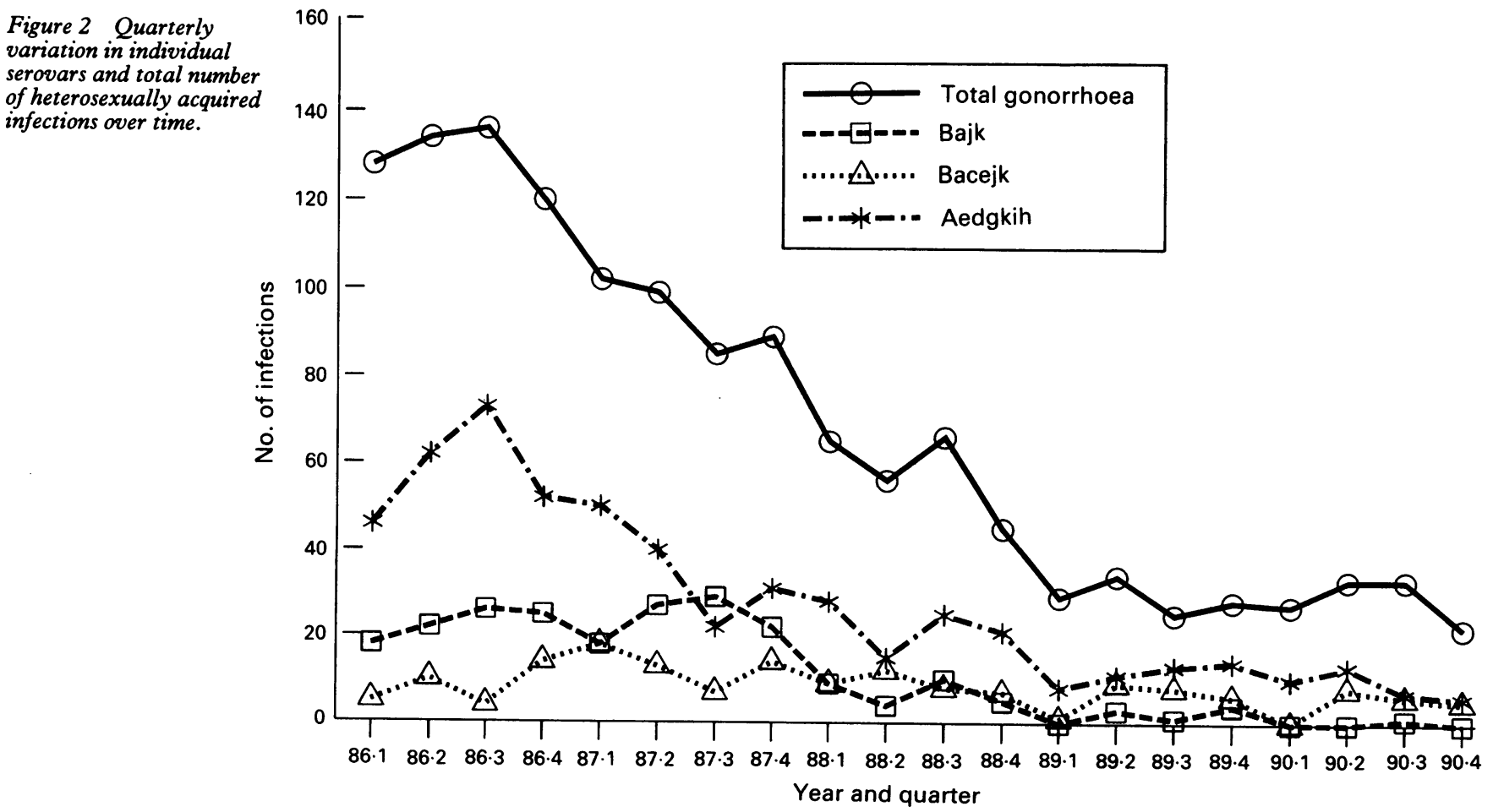

The persistence of certain gonococcal strains with the appearance and disappearance of others over time has been previously described. ${ }^{25}$ Our observed pattern of some strains declining in parallel with the overall trend with infection rate has not, however, been previously described. Possible explanations for these observations must relate either to the serovars themselves or else the population groups which they infect. It is unlikely that any serovar would remain confined to any single group, such as prostitutes, over such a long period although certain serovars are associated with infection in homosexual men. ${ }^{6}$ Alternatively the characteristics of the serovars themselves may explain the observed trends.

Although this study did not look at antibiotic serovars in the same area ${ }^{4}$ reported that Bacejk (1B-1/1B-2) had a higher minimum inhibitory concentration (MIC) for penicillin than average while Bajk (1B-3/1B-6) had a lower than average penicillin MIC. Almost all isolates of Aedgkih (IA-1/IA-2) were highly sensitive to penicillin except for a small number of penicillinase producing strains.

It has been postulated that serovars within any one community die out as protective immunity develops in the population. ${ }^{5}$ It is possible that some strains have a greater ability to evade the immune system than others thus gaining a selective advantage. Although Protein I is relatively less important in the response of antibody in the serum compared with other antigens such as Protein II, pili and lipopolysaccharides it appears to be much more important in the genital antibody response of women compared with the serum antibody response. $^{12}$

A greater variety in the number of different Protein IB serovars was observed than IA strains. This has been noted in other areas ${ }^{45}$ time whilst another persists despite a falling sensitivities, a previous study looking at 
and may be due to a greater potential for antigenic drift in the IB strains compared to the IA strains. ${ }^{13}$ In this population approximately half the infections were due to IA strains and half due to IB strains. It has been observed by others that IB strains tend to be prevalent in large towns whereas IA strains occur more frequently in smaller population centres. ${ }^{14}$

Despite the large fall in the incidence of gonorrhoea over the past 5 years a wide variety of serovars continued to be isolated (Fig 1). It is likely that these minor strains often represent an influx of infections from other geographical areas reflecting the youth and mobility of our population group. It may be that as the overall rate of gonococcal infection falls these new strains are unable to become endemic in the local population and therefore appear only transiently.

The serovar pattern in heterosexuals varies little between men and women (table 3). The observed pattern does, however, differ markedly from that seen in homosexual men from the same area over a similar time period where Ae (IA-4), Back (IB-1/IB-2), Baejk (IB-3/IB-6) and Bacejk (IB-1/IB-2) were the commonest serovars isolated..$^{12}$ This difference in dominant strains between heterosexuals and homosexuals is in agreement with previous studies $^{2}$ and the possible transfer of Bacejk (IB-1/IB-2) between different groups has already been noted.

In conclusion, we have demonstrated a changing pattern of gonococcal serovars over a 5 year period and proposed possible explanations for our observations. Three serovars predominated over the study period with one of these persisting at a constant low level while the others declined in parallel with the overall fall in the incidence of gonorrhoea.

1 Bygdeman S. Polyclonal and monoclonal antibodies applied to the epidemiology of gonococcal infection. In: Young $\mathrm{H}$, McMillan A, eds. Immunological Diagnosis of Sexually Transmitted Diseases. Marcel Dekker, New York 1987: 117-65.

2 Knapp J, King KK, Bonin P, Hook E. Epidemiology of gonorrhoea: Distribution and temporal changes in auxotype/serovar classes of Neisseria gonorrhoeae. Sex Trans Dis 1987;14:26-32.

3 Gill MJ. Serotyping Neisseria gonorrhoeae: a report of the Fourth International Workshop. Genitourin Med 1991;67:53-7.

4 Young H, Moyes A, Robertson DHH, et al. Gonococcal infection within Scotland: antigenic heterogeneity and antibiotic susceptibility of infecting strains. Eur J Epidemiol 1990;6:1-8.

5 Plummer FA, Brunham RC. Gonococcal recidivism, diversity and ecology. Rev Infect Dis 1987;9:846-50.

6 Young H, Moyes A, Ross J, McMillan A. Patterns of homosexually acquired gonococcal serovars in Edinburgh homosexually acquired gonococcal serova

7 Moyes A, Young H. Epidemiological typing of Neisseria gonorrhoeae: a comparative analysis of three monoclonal antibody serotyping panels. Eur J Epidemiol 1991;7: 311-9.

8 Kohl PK, Ison CA, Danielsson D, Knapp JS, Petzoldt D. Current status of serotyping of Neisseria gonorrhoeae. Eur $J$ Epidemiol 1990;6:91-5.

9 Carne CA, Weller IVD, Johnson AM, et al. Prevalence of antibodies to human immunodeficiency virus, gonorrhoea rates and changed sexual behaviour in homosexual men. rates and changed sex $1987 ; \mathbf{i}: 656-8$.

10 Riley VC. Resurgent gonorrhoea in homosexual men. Lancet 1991;337:183.

11 Ross JDC, McMillan A, Young H. Increasing incidence of gonorrhoea and syphilis in homosexual men in Edinburgh. Communicable Diseases and Environmental Health in Scotland Weekly Report 1991;25(91/04):3-4.

12 Lammel CJ, Sweet RL, Rice PA, et al. Antibody-antige specificity in the immune response to infection with Neisseria gonorrhoeae. J Infect Dis 1985;152:990-1001.

13 Sandstrom E, Tam M, Bygdeman S. Antigenic drift of gonococcal protein I as judged by serovar determination. In: Schoolnick GK ed. The Pathogenic Neisseriae Washington DC, American Society for Microbiology, 1985.

14 Danielsson D, Bygdeman S, Kallings I. Epidemiology of gonorrhoea. Serogroup, antibiotic susceptibility and auxotype patterns of consecutive gonococcal isolates from ten different areas of Sweden. Scand J Infect Dis 1983; 15:33-42. 